\title{
Analysis of Vegetation Resistance Based on Two Typical distribution types in Ecological channel
}

\author{
dong liu ${ }^{1}$, chuan Tang $^{1}$, yu Han $^{1}$, jian Chen $^{1}$, and qing Yang ${ }^{1}$ \\ ${ }^{1}$ Affiliation not available
}

April 27, 2020

\begin{abstract}
This paper studied the potential mechanism of form drag from emergent vegetation by the effective combination of experimental verification and numerical simulation. The rigid cylindrical sticks arranged in the open channel were used to simulate vegetation and experiments on flow velocity distribution characteristics in open channel with different vegetation density and vegetation arrangement forms were carried out. Based on the new expression of hydraulic radius from the recent paper, it indicates the form drag from vegetation is closely related with the wake volume near the vegetation. The relationship between the wake volume and form drag is proposed and verified by different experimental conditions. Based on this relationship, the experimental results show that the resistance form of vegetation in vegetated open channels can be divided into: K-type and D-type based on different sticks arrangement within one vegetation patch. The calculated values and measured values achieve a good agreement under different experimental conditions. Meanwhile, numerical simulation based on SAS turbulence model was conducted and the simulation results also fit well with the experimental values so more complex experimental conditions are simulated by numerical simulation method. The results of this study will provide a theoretical basis for the layout of vegetation in the ecological open channel.
\end{abstract}

\section{Hosted file}

Manuscript.docx available at https://authorea.com/users/315506/articles/445896-analysis-of-vegetationresistance-based-on-two-typical-distribution-types-in-ecological-channel 


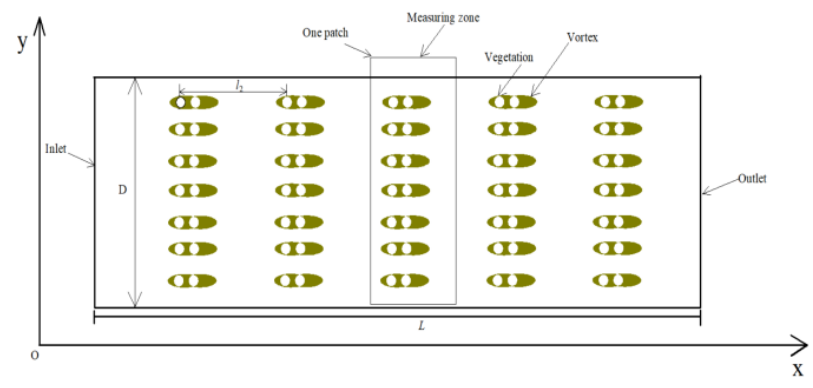

(a) D-type

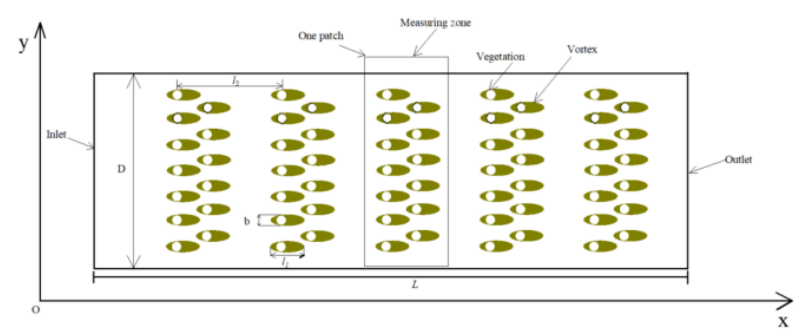

(b) K-type

Fig.1 The schematic of $K$-type and $D$-type vegetation Arrangement 


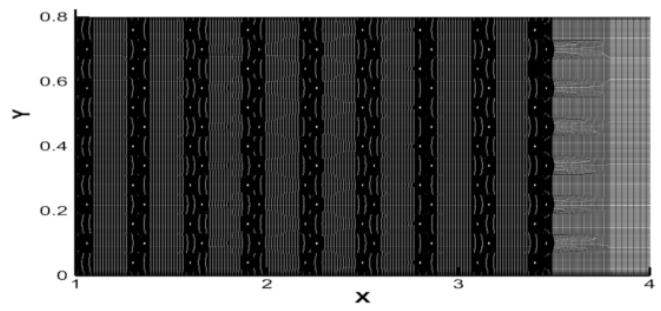

a) Global grid of open channel

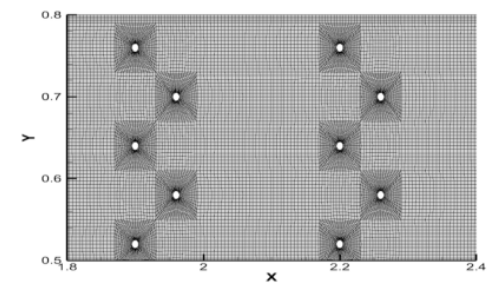

(b) Partial grid of open channel

Fig.2 The mesh structure of vegetated open channel 


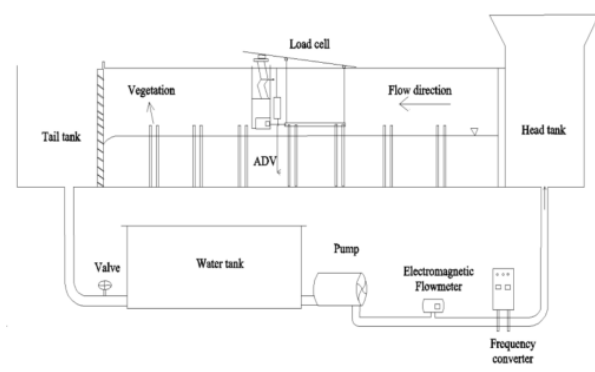

Fig.3 The schematic diagram of the entire experimental equipment

4 


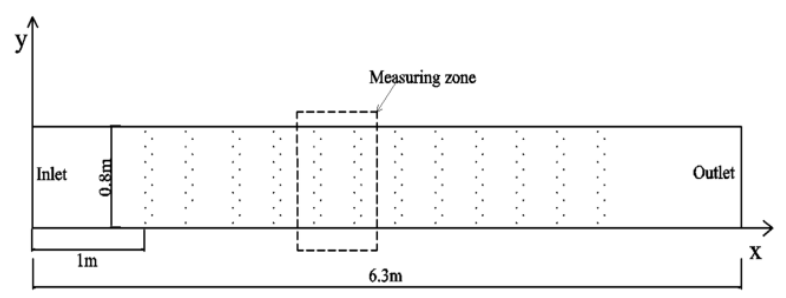

(a)

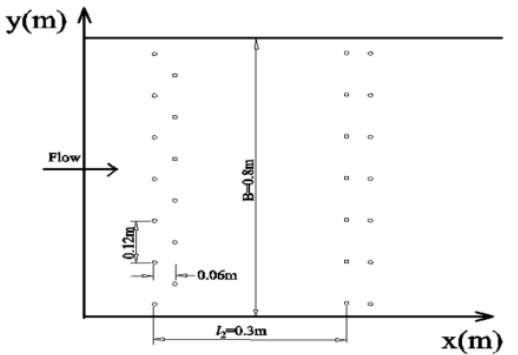

(b)

Fig 4 The schematic diagram of staggered vegetation as a patch: (a) whole schematic diagram; (b) Measuring zone schematic diagram 


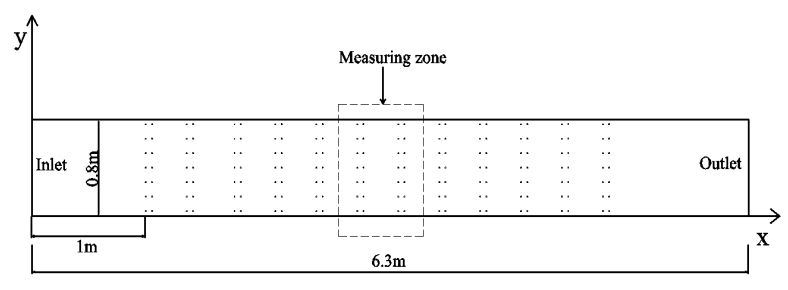

(a)

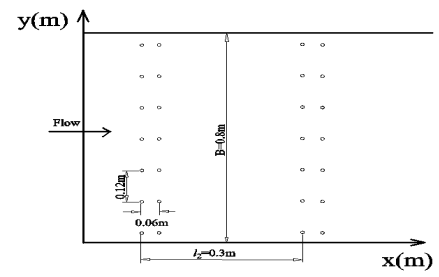

(b)

Fig.5 The schematic diagram of parallel vegetation as a patch: (a) whole schematic diagram; (b)

Measuring zone schematic diagram 


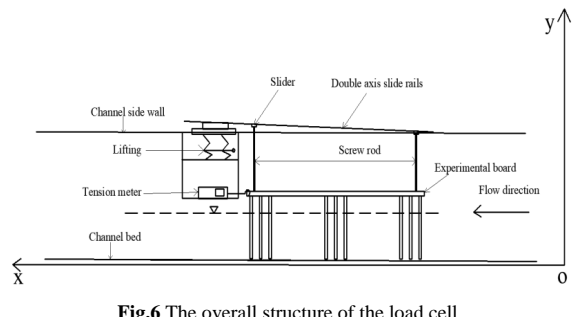

Fig.6 The overall structure of the load cell 


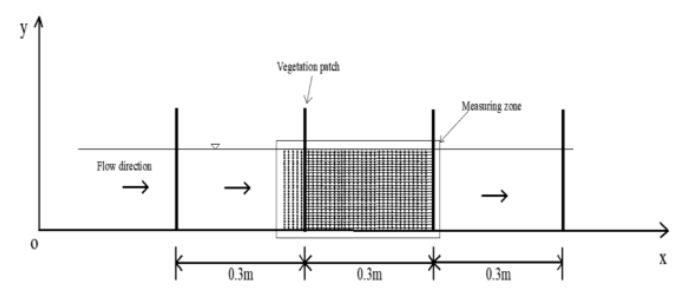

Fig.7 The distribution of the longitudinal measurement point locations 


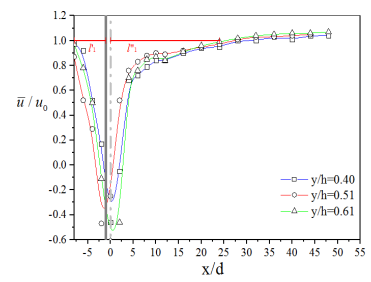

(a) $Q=80 \mathrm{~m}^{3} / \mathrm{h}$

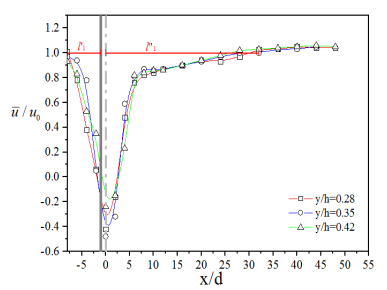

(c) $\mathrm{Q}=100 \mathrm{~m}^{3} / \mathrm{h}$

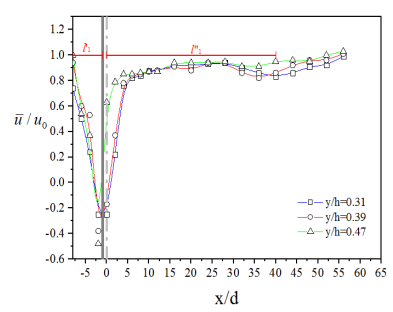

(e) $Q=120 \mathrm{~m}^{3} / \mathrm{h}$

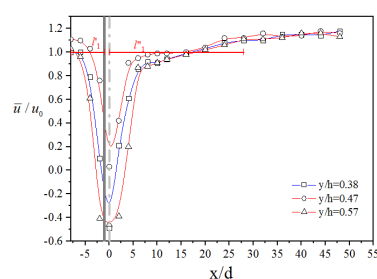

(b) $Q=90 \mathrm{~m}^{3} / \mathrm{h}$

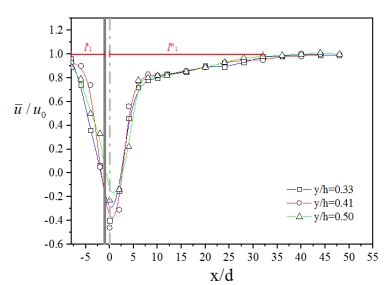

(d) $Q=110 \mathrm{~m}^{3} / \mathrm{h}$

Fig. 8 the longitudinal length of vortex behind vegetation under different flow discharges 


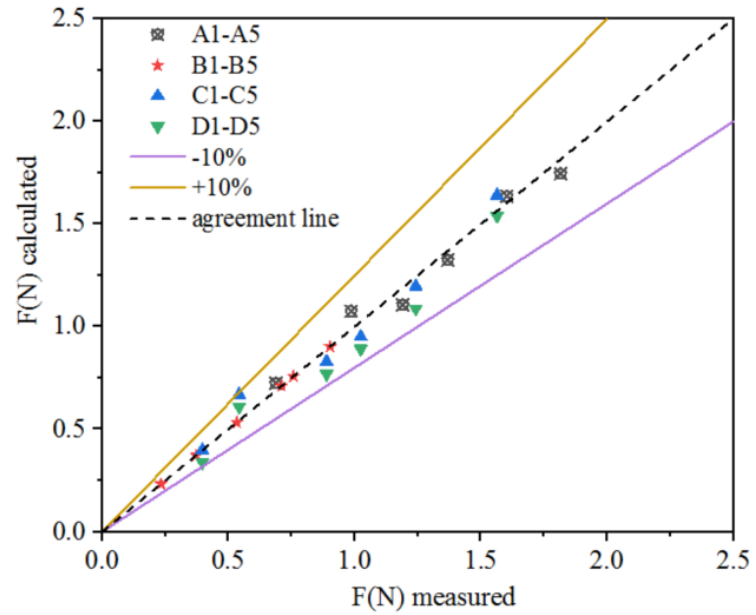

Fig.9 The comparing of measured and calculated form drag values 


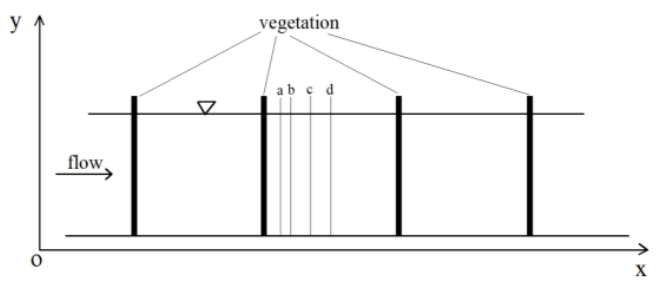

Fig.10 The locations of four different measurement lines 

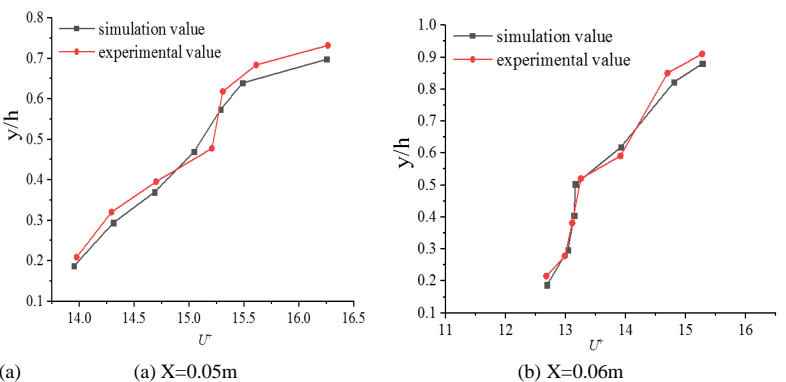

(a)

(a) $\mathrm{X}=0.05 \mathrm{~m}$

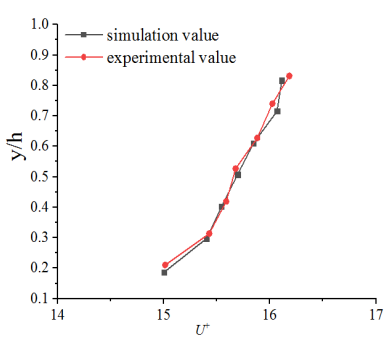

(d) $\mathrm{X}=0.12 \mathrm{~m}$

Fig.11 Comparison of velocity simulation results and measured results on vertical lines at different positions behind patch 


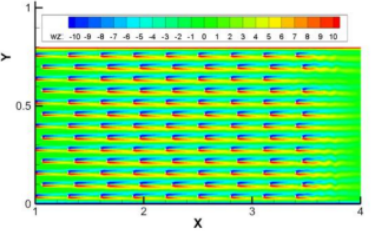

(a) $\mathrm{Q}=90 \mathrm{~m}^{3} / \mathrm{h}$

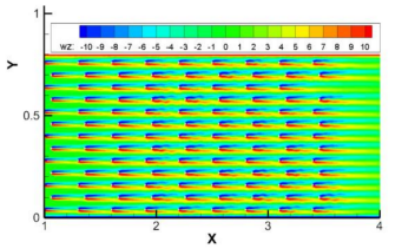

(c) $\mathrm{Q}=110 \mathrm{~m}^{3} / \mathrm{h}$

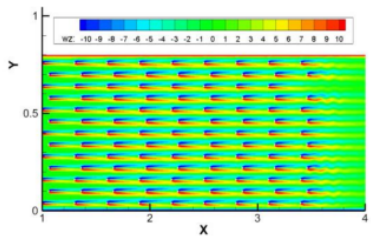

(b) $Q=100 \mathrm{~m}^{3} / \mathrm{h}$

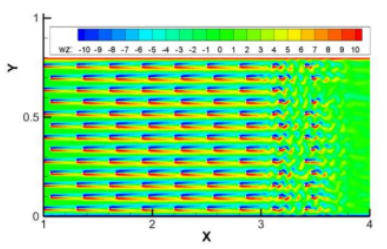

(d) $\mathrm{Q}=120 \mathrm{~m}^{3} / \mathrm{h}$

Fig.12 the development of vortex belts behind the vegetation when two rows of vegetation are in K-type 


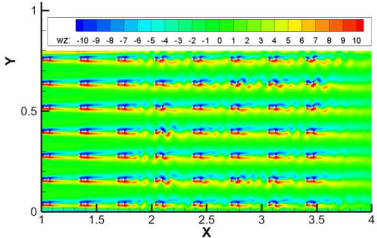

(a) $\mathrm{Q}=90 \mathrm{~m}^{3} / \mathrm{h}$

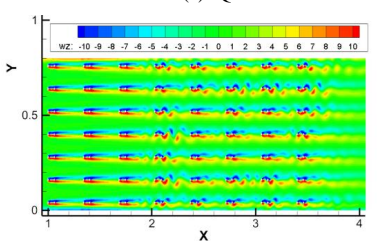

(c) $Q=110 \mathrm{~m}^{3} / \mathrm{h}$

Fig.13 The development of the vortex behind vegetation when two rows of vegetation are in (b) $Q=100 \mathrm{~m}^{3} / \mathrm{h}$

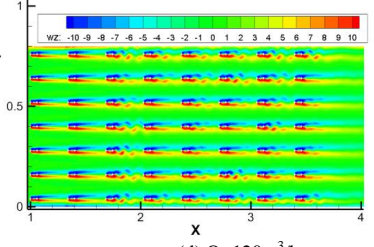

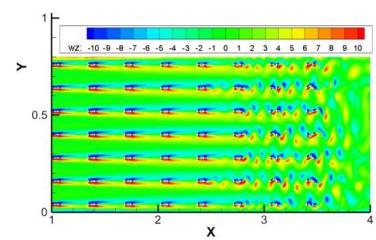


Table 1 Four different vegetation arrangements

\begin{tabular}{|l|l|l|l|}
\hline \multicolumn{1}{|c|}{ Conditions } & $\boldsymbol{l}_{2}(\mathbf{m})$ & $\boldsymbol{N}$ & \multicolumn{1}{c|}{$\boldsymbol{\lambda}$} \\
\hline $\mathrm{A}(\mathrm{A} 1 \sim \mathrm{A} 5)$ & 0.06 & 1 & 0.00285 \\
\hline $\mathrm{B}(\mathrm{B} 1 \sim \mathrm{B} 5)$ & 0.18 & 1 & 0.000953 \\
\hline $\mathrm{C}(\mathrm{C} 1 \sim \mathrm{C} 5)$ & 0.3 & 2 & 0.00098 \\
\hline
\end{tabular}


Table 2 Experimental conditions carried out under each vegetation arrangement

\begin{tabular}{|c|c|c|c|}
\hline Conditions & $\boldsymbol{Q}\left(\mathbf{m}^{\mathbf{3}} \mathbf{h}\right)$ & \multicolumn{1}{l}{$\boldsymbol{h}(\mathbf{m})$} & $\boldsymbol{R} \mathbf{e}$ \\
\hline 1 & 90 & 0.106 & 24703 \\
\hline 2 & 100 & 0.114 & 27021 \\
\hline 3 & 110 & 0.121 & 29324 \\
\hline 4 & 120 & 0.128 & 31627 \\
\hline
\end{tabular}

